\title{
ENFOQUE EPISTEMOLÓGICO \\ PARA EL ESTUDIO \\ DE LAS FORMACIONES SOCIALES \\ CAPITALISTAS SUBDESARROLLADAS ${ }^{1}$
}

\author{
Xabier Arrizabalo Montoro2
}

1

l presente artículo inicia una serie de tres en los que vamos

a discutir acerca de subdesarrollo desde una perspectiva eco nómica. Más concretamente, intentaremos avanzar algunos elementos para el estudio del subdesarrollo en la actualidad. En este primero, pretendemos efectuar una aportación a los fundamentos y metodología de análisis, es decir, al enfoque epistemológico para su estudio. En los dos siguientes abordaremos, respectivamente, una aproximación teórica y una aproximación al contexto histórico actual en que tiene lugar el desempeño de las economías subdesarrolladas.

En este artículo presentaremos un apartado de carácter introductorio. A continuación, planteasemos una crítica a la aplicación de las teorías "convencionales" a este tema. En el tercer apartado, discutiremos las diferencias existentes entre las distintas posiciones "no convencionales". A partir de ello, concluiremos exponiendo nuestra posición epistemológica.

\section{Introducción}

T primero que hay que hacer es, lógicamente, explicar qué es Lel subdesarrollo. Para ello, empezaremos planteando el significado de la existencia de formaciones sociales. La categoría modo 
de producción capitalista no toma concreción en sí misma sino que lo hace, históricamente, en realidades sociales específicas. El análisis comparativo de estas distintas concreciones - las formaciones sociales- pone en evidencia niveles de desarrollo cuantitativamente distintos que se tornan en diferencias cualitativas.

Con base en ello, se propone una tipología de formaciones sociales capitalistas que las agrupa en desarrolladas o subdesarrolladas 3 . De manera que, si bien el elemento central para el análisis del capitalismo es el estudio de la relación capital-trabajo, la mejor forma de llevar a cabo cierto tipo de particularización de dicho análisis -como la que nos ocupa- es a través de la unidad de análisis formación social. Lo cual no presupone ningún tipo de posicionamiento respecto de su pretendida homogeneidad sino justamente al contrario: se le considera un ámbito más, crucial, donde toman concreción las principales contradicciones, y, más específicamente, la del antagonismo entre clases - si se prefiere, la lucha de clases-.

A partir de lo anterior se puede esbozar una primera aproximación al fenómeno del subdesarrollot, en términos comparativos. Así, hablaremos de subdesarrollo para referimos a las formaciones sociales caracterizadas por la existencia de una serie de indicadores, descriptivos, que presentan niveles cuantitativamente distintos a aquellos presentes en lo que denominaremos formaciones sociales capitalistas desarrolladas (FSCD) - por oposición a las formaciones sociales capitalistas subdesarrolladas (FSCS) 5 - A modo de ejemplo de indicadores, pueden citarse, entre muchos otros, los siguientes: nivel de renta per capita, tasa de alfabetización, medida de esperanza de vida, índice de satisfacción de necesidades básicas (nutrición, vestido, sanidad, educación, vivienda, etcétera), mutuamente interrelacionados entre sí. Junto a ellos, en un nivel explicativo mayor, es decir, no manifestación última del subdesarrollo como éstos, pero tampoco en el plano explicativo fundamental, se encontrarían otros elementos como las bajas tasas de ahorro, los reducidos niveles de inversión productiva, la escasa existencia de personal cualificado, la estructura de demanda distorsionada, etcétera. 
En todo caso, tanto unos como otros no son sino manifestaciones -o expresiones - de un conjunto de interrelaciones entre distintos elementos internos y factores externos que configuran una determinada estructura económica que afecta tanto a la esfera productiva como a la circulatoria. Conjunto de interrelaciones que constituyen el primer acercamiento a la conceptualización del subdesarrollo, no en cuanto a sus indicadores sino a su esencia. Sobre ello se volverá más adelante ya que lo importante en este momento es plantear cómo las diferencias cuantitativas se transforman, histórica y dialécticamente, en cualitativas. Dicha transformación, que lleva a la existencia de una situación cualitativamente distinta, es la que nos permite hablar de formaciones sociales capitalistas subdesarrolladas y desarrolladas. Distinción que queda subordinada, en todo caso, a la preeminencia de lo común en ambos tipos de sociedades que es su conformación capitalista.

Es fundamental reiterar que todo lo expuesto no implica considerar a cada formación social específica como una unidad de análisis homogénea ya que, como se recalcará más adelante, el análisis en términos de clase supone para nosotros una de las referencias teórico-metodológicas básicas.

El enfoque epistemológico y la posiçón teórica más adecuados para el análisis de las formaciones sociales capitalistas subdesarrolladas ha sido motivo de arduas discusiones. Piénsese que la considerasión de éstas como objeto de estudio específico, valorando su peculiaridad, es relativamente reciente. La polémica adquiere relieve, especialmente, desde los años 50 y 60 con la irrupción en el debate de elaboraciones teóricas de científicos sociales procedentes de FSCS, mayormente latinoamericanos. Precisamente estos planteamientos surgen como reacción ante la incapacidad manifiesta de trasladar con éxito a estas realidades los métodos de análisis paradigmáticos en las FSCD. Recuérdese que dichos esquemas imperantes han venido siendo, en función del momento concreto, los asociados a la escuela clásica, neoclásica o keynesiana6. 
2 Crítica a la aplicación de los enfoques "convencionales" al estudio del subdesarrollo

T a crítica a la aplicación de las teorías convencionales - básica se basa en una serie de aspectos centrales que serán tratados a continuación y que, sustancialmente, son comunes a ambos enfoques?.

El primer elemento es el del horizonte parcial y superficial que se adopta respecto del fenómeno del subdesarrollo. Parcial porque se elaboran análisis "puramente" económicos, de carácter eminentemente descriptivo y cuantitativista que dejan de lado importantes aspectos asociados a las estructuras sociales y políticas -entre otras- de estos países. Superficial porque se sitúa el análisis en el plano de las expresiones o manifestaciones del subdesarrollo sin ahondar en sus causas y explicaciones, en su esencia. De esta manera, se tiende a identificar los conceptos de crecimiento -e incluso industrialización-y desarrollo. Frente a ello, resulta necesaria una perspectiva global y totalizante que comprenda las distintas dimensiones de los fenómenos sociales, así como sus mutuas interrelaciones. Esto no significa, obviamente, que no deban realizarse investigaciones desde la disciplina, en el caso, de la economía, sino que ellas deben ser contextualizadas atendiendo a los distintos aspectos de la realidad social que influyen y condicionan los aspectos propiamente económicos 8 .

El segundo elemento dice relación con el carácter ahistórico que presenta el pensamiento convencional. Tiene especial significación por cuanto que supone obviar el importante papel que juega la expansión del modo de producción capitalista en la conformación de la situación crónica y estructural de subdesarrollo en estos países. Además ello plantea un determinismo tal que hace conceptualizar al subdesarrollo como una mera etapa "necesaria", "ineludible" o "inevitable" en el camino hacia el desarrollo9. Sin embargo, la condición de historicidad (que no historicismo determinista), inherente a todos y cada uno los fenómenos sociales implica la imprescindible consideración de su evolución en el tiempo, en tanto que condicionante básico de su conformación actual, así como de su posible desempeño a futuro. 
El tercer elemento lo constituye el carácter lógico-formal, mecanicista o causalista que impregna estos enfoques. Llevan a cabo estudios descriptivos, meramente formales, en los que el argumento general es reducible, en la mayor parte de los casos, a cuestiones estrictamente cuantitativas. La no consideración $\longrightarrow$, directamente, la negación- del conflicto de clase como motor de la historia, les permite obviar la principal contradicción existente en el modo de producción capitalista. Opuesto a este tipo de planteamientos lineales aparece la necesidad de un enfoque de tipo dialéctico que atienda al papel crucial que desempeña la contradicción, considerando la transformación de cambios cuantitativos en cualitativos que interrumpen la gradualidad y propician las transformaciones a través de saltos.

Finalmente, el cuarto elemento es el sustrato idealista sobre el que descansan estos enfoques. En efecto, pretenden explicar los fenómenos remitiendo a factores ajenos a la propia realidad que se trata de interpretar, factores situados en el terreno de las ideas puras, desligadas de la realidad. Con ello se elude el tratamiento de los factores que están en la raíz de dichos fenómenos, lo que permite, de nuevo, obviar aquellos aspectos susceptibles de cuestionar las bases mismas de reproducción capitalista. En contraposición a lo anterior, nosotros nos decantamos por un enfoque materialista que aborde la explicación de la realidad a partir de la comprensión de los elementos que conforman la propia realidad sin aludir a factores ajenos a ella. (Para evitar confusiones y a modo de ejemplo, aclaramos que, obviamente, las relaciones exteriores en una economía no son ajenas a dicha economía sino que forman parte integrante de ella, de forma consustancial) 10.

Por tanto, epistemológicamente, los enfoques convencionales adoptan una posición dogmática. De manera tal que en su análisis no hay espacio para la especificidad de cada situación concreta, definida por lo común y lo peculiar que toma en su caso la interrelación de los distintos factores - no sólo económicos- que explican su realidad. Nosotros sugerimos un enfoque crítico tanto respecto del convencional como respecto a la aplicación de recetas preelaboradas cualesquiera que sean éstas. Lo cual no pretende implicar un empirismo vulgar, dado que, en todo caso, reclamamos como necesario un punto de partida basado en la adopción 
de una serie de supuestos teóricos. Crítico, también, en el sentido de la incorporación de las aportaciones o elementos positivos que puedan eventualmente incorporar otros enfoques -incluidos los examinados-.. Y crítico también, finalmente, en cuanto a la lectura que sugerimos de cualquier texto, por supuesto que éste incluido.

Como plantea Ramos), "en resumen este enfoque convencional es inadecuado, en mi opinión, para abordar el subdesarrollo, en cuanto no intenta un análisis completo del fenómeno, no lo sitúa históricamente y no penetra en los mecanismos internos que lo explican y lo reproducen".

Antes de concluir el presente epígrafe, queremos realizar una última aclaración que resulta central. Ni todos los autores o corrientes encuadrados dentro de lo que hemos denominado enfoque convencional aceptan "disciplinadamente" todos los rasgos, ni todos los autores y corrientes dentro de lo que sería el enfoque crítico rompen necesariamente con cada uno de los rasgos citados - tema que será levemente abordado en el epígrafe siguiente-. De ahí que la heterogeneidad sea enorme, no sólo entre escuelas, sino también al interior de éstas. Un análisis pormenorizado de cada una de ellas excede en mucho el alcance del presente trabajo y, además, carece de sentido en relación al mismo. Lo que nos interesa es, no tanto la presencia o ausencia de uno o más rasgos determinados en cada autor o corriente, sino el enfoque y método concreto que plantean a partir de su interrelación. $Y$ más concretamente, detallar el que constituye nuestra referencia para el conjunto de la investigación.

\section{Los enfoques "no convencionales": elementos comunes y ele- mentos divergentes}

Tna vez planteada la crítica a la aplicación de las teorías conde método son apropiados al fin de encarar el objeto de estudio que manejamos.

En los párrafos anteriores ya hemos esbozado los elementos con que debe contar dicho enfoque. Así, sintetizando lo expuesto, éste debe ser global y totalizante, histórico, materialista y dialéctico. 
A lo cual habría de añadírsele el de crítico respecto de las formas paradigmáticas y dogmáticas de análisis del fenómeno del subdesarrollo.

Estos elementos -que nosotros consideramos necesarios para la conformación de un enfoque correcto- aparecen, en muy distintos grados, en parte importante de los estudios sobre el subdesarrollo que emergen en las décadas de los 50 y de los 60 , especialmente procedentes de América Latina, dentro de lo que Ramos caracteriza, con demasiada amplitud, como "enfoque dialéctico-estructural" -que sería la agregación de los enfoques dialécticos y los estructurales-, incluyendo desde los de la Comisión Económica para América Latina -actualmente, ampliada a "y el Caribe" para incluir a los países de este área- (CEPAL) hasta los explícitamente marxistas, pasando por los encuadrables en el enfoque dependentista. Por supuesto, dentro de esta amplísima categoría "dialéctico-estructural" se encuentran diferencias importantísimas entre las distintas posiciones existentes en su seno, lo cual amerita que nos detengamos algo más en este punto.

El planteamiento cepalino, dentro del cual -por tomar alguna referencia de autor- nos referiremos al "ortodoxo", expuesto por Prebisch ya desde los primeros documentos de la Comisión, incorpora parcialmente algunos de los elementos seleccionados por nosotros como necesarios. Efectivamente, es un planteamiento crítico de la teoría del comercio internacional convencional. Incluye aspectos no directamente económicos (no tanto en los primeros momentos cuanto años después; si bien, en todo caso, siempre aparecen, al menos, algunos componentes de la estructura social y politica completando y matizando el análisis estrictamente económico así como análisis que van más allá del tratamiento de las manifestaciones del subdesarrollo, indagando en sus causas - mas no en las últimas-). Y también plantea un enfoque histórico analizando la evolución en el tiempo de la modalidad de inserción en la economía mundial de estos países. Pero se conforma con plantear un análisis de las relaciones entre variables calificable como lógico-formal y no dialéctico. No plantea como elemento crucial la existencia de contradicciones inherentes a la evolución de estas sociedades, asociadas a conflictos tanto externos como internos, derivadas de su conformación como capitalistas. Únicamente su- 
giere la presencia de aspectos de desigualdad y asimetría en la relación entre las FSCD ("centro" en su terminología) y las FSCS ("periferia"). Pero entendidos como cuestiones que son subsanables, mediante determinadas transformaciones (desde la famosa y tradicional "industrialización por sustitución de importaciones" hasta la actual "transformación productiva con equidad"), propugnando, con ello, la viabilidad de un desarrollo nacional capitalista autónomo y autocentrado. Asimismo, este enfoque presenta una dimensión idealista y no materialista en la medida en que se elude referir la problemática del subdesarrollo latinoamericano a los elementos esenciales de la economía política del capitalismo. Dicho de otro modo, más sencillamente, el obstáculo central con el que choca esta corriente es que no parte de un enfoque de "economía política", sino que se sitúa en la discusión, parcial y superficial, de la "política económica. Es oportuno señalar aquí que esta posición es compartida, en lo sustancial, por una parte de los autores ubicados dentro del enfoque dependentista11.

Otra parte de estos autores dependentistas, sitúan la cuestión central -como su propia denominación indica- en la dependencia (comercial, financiera, tecnológica, de patrones de consumo, etcétera) de las FSCS respecto de las FSCD -con diferentes terminologías para los tipos de países como las de "satélite" y "metrópoli", etcétera-. Si bien se puede aceptar que ello constituye un intento dialéctico por la existencia de conflicto entre contrarios, entendemos que elude el tema de la propia esencia del modo de producción capitalista a escala mundial y en cada formación social. Este no es otro que la presencia de la contradicción en términos de clases antagónicas, por el papel relativo que juegan en las relaciones de producción. De manera que, si bien el carácter dependiente de estas sociedades es importante, éste no es el elemento central. Esto será discutido con mayor detenimiento en los siguientes epígrafes de este artículo.

En todo caso, debe señalarse que la heterogeneidad existente entre los distintos autores que hacen un análisis dependentista, obligaría a tratarlos con un grado de desagregación mayor - prácticamente, autor por autor-. De manera que lo expuesto hasta ahora es solamente una aproximación muy abstracta a lo que representan algunas líneas dentro de las distintas existentes suscep- 
tibles de ser calificadas como dependentistas.

A pesar de que, bajo nuestro punto de vista, ni el enfoque cepalino, ni el dependentista -en sus distintas formulacionesson los más adecuados para abordar el estudio de la realidad de las FSCS, sí se les debe reconocer una serie de aportaciones tanto metodológicas como de contenido a dicho estudio, especialmente de algunos autores "dependentistas" como Marini o Quijano Obregón. Aportaciones que, por nuestra parte, no dudamos en integrar a nuestras investigaciones.

4 La necesidad de un enfoque crítico, histórico, materialista, dialéctico y totalizante: la referencia marxista

A partir de lo expuesto hasta el momento, resulta evidente nues mo como teoría de referencia para el estudio de la problemática de los países subdesarrollos - así como del desarrollo-, recogiendo, sin duda alguna, toda aportación procedente de otras posiciones teóricas que, efectivamente, contribuya a un mejor acercamiento al conocimiento de la realidad12. Al respecto corresponde realizar algunas precisiones.

Tradicionalmente ha habido una crítica a la teoría marxista en cuanto a su aplicabilidad para el estudio del fenómeno del subdesarrollo. Consiste en su presunta incapacidad para ello por el "error" del propio Marx - a mediados del siglo pasado- al comentar que "el país industrialmente más desarrollado no hace sino mostrar al menos desarrollado la imagen de su propio futuro".

Por una parte, es cierto que Marx no lleva a cabo un análisis expreso de la peculiaridad del desarrollo capitalista causado por la penetración extranjera respecto del originado endógenamente salvo en varios casos particulares (como la India o Irlanda). Pero es esencial aclarar algunos puntos que permitan entender por qué no lo hace así y su significado. En primer lugar, el mismo autor matiza que el horizonte de su obra se relaciona con el desarrollo histórico europeo, advirtiendo, en una carta enviada a un periódco socialista ruso en $1877-6$ años antes de su muerte-, que no se quiera "convertir mi esbozo histórico sobre los origenes del capitalismo en la Europa occidental en una teoría filosófico-histónica sobre la trayec- 
toria general a que se hallan sometidos fatalmente todos los pueblos, cualesquiera que sean las circunstancias históricas que en ellos concurran"13. Como expone Palma, "la enorme importancia de estas afirmaciones (...) consiste en mostrar que él no vio la historia como una secuencia mecánica de etapas predeterminadas por las cuales toda sociedad está condenada a pasar, sino como un proceso en el cual la particularidad de cada situación histórica tiene un importante papel que desempeñar"14. De manera que la frase citada hay que entenderla en cuanto a la extensión y consolidación hegemónica de la lógica capitalista en estas partes del mundo, lo cual resulta, globalmente, indudable.

Ello se aprecia más claramente si precisamos al carácter histórico genéricamente referido anteriormente, con la cualidad de materialista, es decir, remitiéndonos al materialismo histórico. Concretado en la propuesta de método capaz de interpretar la realidad a partir de las condiciones materiales concretas -rasgo igualmente asociado al carácter dialéctico del método; i.e.: el materialismo dialéctico- 15 .

Trotski profundiza a Marx en este sentido, cuando escribe que "el capitalismo prepara y, hasta cierto punto, realiza la universalidad y permanencia en la evolución de la humanidad. Con esto se excluye ya la posibilidad de que se repitan las formas evolutivas en las distintas naciones. Obligado a seguir a los países avanzados, el país atrasado no se ajusta en su desarrollo a la concatenación de las etapas sucesivas. (...) el desarrollo de una nación históricamente atrasada hace, forzosamente, que se. confundan en ella, de una manera característica, las distintas fases del proceso histórico. Aqui el ciclo presenta, enfocado en su totalidad, un carácter confuso, embrollado, mixto. (...) las leyes de la historia no tienen nada en común con el esquematismo pedantesco. El desarrollo desigual, que es la ley más general del proceso histórico, no se nos revela, en parte alguna, con la evidencia y la complejidad con que la patentiza el destino de los paises atrasados" 16 .

Por ello, la famosa y extendida crítica a Marx por la idea que hemos comentado, hay que relativizarla entendiendo dicha idea, correctamente, en el sentido de la tendencia de estos países a conformarse como formaciones sociales de tipo capitalista $\longrightarrow$ formaciones sociales en las que predomina el modo de producción capitalista por sobre otros igualmente vigentes-, tendencia que históricamente se ha visto contrastada. 
Desenmarañado el "error" —que debe su profusión tanto a la posición estalinista que induce al mismo por su lectura sesgada de Marx, como a la interesada interpretación de sectores intelectuales de la burguesía "nacionalista" latinoamericana que rechazan las teorías que afectan a sus intereses, tildándolas de "inaplicables" por ser procedentes de otras realidades-, queda aclarado que se le "puede" reprochar a Marx el no haber estudiado la realidad de los países "menos avanzados" — en la misma medida que pueda reprochársele a cada autor todo aquello que no ha estudiado-, pero no haber obviado, o negado, su peculiaridad.

A continuación, efectuaremos otra puntualización importante. La obra de Marx presenta varias dimensiones. Una, lleva a cabo la interpretación del modo de producción capitalista en el plano teórico. Otra, sobre la base de dicha interpretación, realiza el análisis concreto del desarrollo de dicho modo de producción en el plano aplicado, para el caso europeo. No se trata aquí de efectuar una disertación sobre las distintas interpretaciones de la obra de Marx, sino de aclarar en que sentido es en el que hablamos de "referencia marxista" en este caso.

Así, concretamente, es la primera de las dos dimensiones citadas la que interesa a los efectos de este artículo. Efectivamente, consideramos su establecimiento de categorías y conceptos fundamentales y sus criterios metodológicos sugeridos como los más adecuados instrumentos para el estudio de una formación social capitalista, como que la que nos ocupa en el presente trabajo17.

Ahora bien, ello no supone un marco teórico cerrado que guía cada paso en el proceso de investigación, sino un referente "filosófico" (sustentado en la consideración acerca del papel que juega la lucha de clases - cuya concreción en el modo de producción capitalista es la oposición capital/trabajo- como motor de la historia) para el encuadramiento del análisis. Razón por la cual, de lo que se trata no es de encajar forzosamente el objeto concreto de estudio en las "leyes" del desarrollo del capitalismo, sino que éstas sirvan como tal referente. Como exponía Engels en 1895, "toda la concepción de Marx no es una doctrina, sino un método. No ofrece dogmas hechos, sino puntos de partida para la ulterior investigación, y el método para dicha investigación"18. 
Comoquiera que el marxismo no lo percibimos como una estructura cerrada y determinista, lo que planteamos no es una aplicación milimétrica de una receta determinada, sino contar con él como referente teórico último. Naturalmente, esto también se relaciona con las variables e indicadores a utilizar. Normalmente, pretender la utilización lineal de las categońas y conceptos marxistas al pie de letra requeriría un material estadístico que no suele estar disponible porque, sencillamente, no existe. Construirlo llevaría un trabajo de elaboración estadística de inalcanzable envergadura que, además, en muchas ocasiones, nos alejaría tanto de los objetivos como del alcance de nuestra investigación. Pero además de esta imposibilidad, pensamos que no es necesaria la utilización escrupulosa de indicadores ajustados cuidadosamente a los mencionados conceptos y categorías $\longrightarrow$ variables - sino que estos pueden perfectamente aparecer como referentes últimos en cuanto a grandes tendencias - sin que haya un tratamiento estadístico pormenorizado de los mismos. No obstante, no sólo no desalentamos esta tarea de construcción y adaptación de indicadores sino que abogamos por ella.

Como colofón de este apartado podemos concluir, muy sintéticamente, diciendo que adoptamos un marco teórico de referencia de carácter marxista porque entendemos que es el que mejor nos posibilita el acercamiento al conocimiento de nuestro objeto: una formación social capitalista.

\section{Recopilación}

T os puntos que siguen recopilan, muy sintéticamente, las pau $\downarrow$ tas básicas del enfoque epistemológico que hemos expuesto en el presente artículo.

1. Las teorías convencionales - básicamente, keynesiana y neoclásica- no son válidas para efectuar una aproximación analítica e interpretativa del fenómeno del subdesarrollo - ni del desarrollo- por abordarlo desde un enfoque epistemológico de carácter dogmático, parcial-superficial, ahistórico, lógico-formal-mecanicista e idealista.

2. En contraposición a ellas, proponemos un método para abordar su estudio que parta de un enfoque epistemológico crítico, 
global y totalizante, histórico, materialista y dialéctico.

3. Dentro de las posiciones críticas respecto a la validez de las teorías convencionales, el punto que supone la línea de fractura entre ellas es el que las divide en función de la adopción del análisis materialista y dialéctico como una de las referencias epistemológicas fundamentales. Esto es así por cuanto entendemos que el elemento central para el análisis debe ser la consideración de la existencia de clases antagónicas. A partir de ello, el enfoque por el que se opta es encuadrable en el marco genérico del marxismo, por ser el que mejor nos posibilita el acercamiento al conocimiento de una formación social capitalista, caracterizada, precisamente, por la mencionada existencia de clases antagónicas.

\section{Referencias bibliográficas}

- CUEVA, Agustín; Problemas y perspectivas de la teoría de la dependencia, en Debate sobre la teorla de la dependencia Editorial EDUCA, San José, Costa Rica, 1979.

- DABAT, Alejandro; El mundo y les naciones, UNAM, Cuernavaca, 1993.

* GILL, Louis; Économie mondiale et impérialisme, Boréal Express, Québec, 1983.

* GRIFFIN, Keith, y GURLEY, John; "Análisis radicales del imperialismo, el Tercer Mundo y la transición al socialismo: una panorámica”, Información Comercial Española agosto/septiembre 1986, págs. 229 a 273.

- MARX; Karl; El Copital (Crítica de la Economía Política), Siglo XXI, 8 vols., Madrid, 1984; primera edición de la traducción: Buenos aires, 1975.

- PALAZUElOS, Enrique (ed.); Las economías capitalistas durante el periodo de expansión 1945-1970, Akal, Madrid, 1986.

- PALMA, Gabriel; Dependencia y desarrollo: uno visión crítica en SEERS, Dudley (comp.); La teoría de la dependencia (una revaluación crítica) FCE, México, 1987.

- PAZ, Pedro; El enfoque de la dependencia en el desarrollo del pensamiento económico latinoamericano, México, 1981, págs. 61 a 81

- RAMOS, Antonio; El subdesarrollo: introducción metodológica, mimeo, UCM 1979.

- _; Las fases de expansión del MPC, mimeo, UCM 1980.

- _- Las consecuencias de la expansión copitalista: la formación del subdesarrollo, mimeo, UCM 1980.

- _- Sobre la reproducción del subdesarrollo, mimeo, UCM 1980.

- SUNKEL, Osvaldo y PAZ, Pedro; El subdesartollo latinoamericano y la teoría del desarrollo, Siglo XXI, México, 1970.

- VALENZUELA F., José; ¿Qué es un patrón de acumulación?, Facultad de Economia, UNAM, México, 1990.

- _-; Crítica del modelo neoliberal, Facultad de Economía, UNAM, México, 1991. 
Notas:

1. El presente artículo se basa en el capítulo II de ARRIZABALO, Xabier; Transnacionalización y subdesarrollo: Chile, 1973/1990 (Resultados económicos y significado histórico de la dictadura y el neoliberalismo), Tesis Doctoral, Madrid, 1993.

2. Profesor de Economía de la Universidad Complutense de Madrid.

3. La utilización del término "subdesarrollo" ha sido - y sigue siendo- motivo de arduas discusiones que, ciertamente, exceden a una cuestión meramente terminológica. Efectivamente, la utilización de uno u otro término supone, implícitamente, una posición de "contenido" al respecto. Así, se habla de "periferia" - por oposición a "centro"- en la obra de Prebisch. De "formaciones de transición bloqueada" en propuesta de Amin. De "dependientes", por parte de muchos autores encuadrados -o encuadrables- en el enfoque de la dependencia. Y un largo etcétera -en el que se incluirían, también, expresiones como "subdesarrolladores" para referirse a los palses que nosotros estamos denominando "desarrollados"- - Sin entenderlo como una cuestión central, pero sí significativa, asumimos el término "subdesarrollo" como expresión de una gradación - no tanto cuantitativa cuanto cualitativa - en el desarrollo de estos países respecto de los que efectivamente están más "desarrollados", considerando, en todo caso, el nivel de abstracción que ello implica. De manera que esto no supone -ni mucho menos- considerar el subdesarrollo como una etapa necesaria e inevitable hacia el "desarrollo" en la forma en que tiene lugar en los que llamamos "desarrollados", ni siquiera -cuestión importante- que en estos se pueda hablar estrictamente de desarrollo sino que sólo haciéndolo relativo a aquellos.

4. En todo este articulo nos referimos al subdesarrollo tomando como referencia los rasgos de lo que sería un país subdesarrollado "típico" o "promedio". Como se sabe, la heterogeneidad entre los distintos países - como en su interior - es enorme. No obstante, conscientes de ello, nos conformamos en este caso con centramos exclusivamente en lo común.

5. Resulta obvio que en este tipo de formaciones sociales se da la convivencia de distintos tipos de modos de producción, de la misma manera que, históricamente - $y$ actualmente, si bien en infima medida- en las desarrolladas. Cuando se habla de "capitalista", nos referimos al modo de producción dominante que subordina $-e$ incluso hace funcional- a los otros que puedan eventualmente existir, tanto al nivel de cada formación social como a escala mundial.

6. La afirmación de que dichos cuerpos téricos no son correctos para su aplicación con éxito al análisis de las FSCS no implica que, contrariamente, sí sean adecuados para el de las FSCD. Si bien la argumentación de ello excede el alcance del presente trabajo, nuestra posicion al respecto es que tampoco lo son para éstas. Es decir, el problema no radica - como sostienen algunos investigadores - en que teorías válidas para el estudio de países desarrollados no lo sean para los subdesarrollados por no considerar la peculiaridad de éstos, sino que tampoco lo son en aquellos. No obstante, existe un elemento específico -ciertamente relevante- de su no aplicabilidad para el caso de las FSCS: radica en que ni siquiera, salvo en pocas excepciones, son 
consideradas como objeto de estudio específico.

Por otra parte, no debe olvidarse el hecho de que la escuela paradigmática ha sido siempre aquella cuyas recomendaciones de política coincidieran con los intereses del capital hegemónico del período histórico de que se tratara. Lo cual es aplicable tanto a la clásica, como a la neoclásica, como a la keynesiana - consideradas, en todo momento, desde una perspectiva genérica- Es decir, que la cuestión de que hablamos no es aséptica, sino que tiene que ver directamente con la relación de fuerzas existente en el terreno politico, tanto del lado del capital como del trabajo. Relación de fuerzas de la que los distintos paradigmas teóricos son expresión e instrumento.

7. Para la crítica de la aplicación de las teorías convencionales al estudio de las FSCS, nos basamos en gran medida en los trabajos de RAMOS (1980) y de SUNKEL y PAZ (1970), citados en la bibliografía. Por cierto que una parte importante de estos comentarios son perfectamente válidos para cuando dichas teorías son aplicadas al análisis de las FSCD. Conviene aclarar que la crítica que se efectúa en este apartado no se lleva a cabo en cuanto a la discusión de las distintas escuelas, sino a los elementos centrales comunes a ellas, siendo conscientes, en todo caso, de las importantes diferencias existentes entre ambas.

8. Todo ello no implica la inexistencia de gradación en la importancia relativa entre los fenómenos "correspondientes" a las distintas ramas de las ciencias sociales, dado que efectivamente el sustrato económico juega un papel preponderante. Lo que se pretende señalar pues, es que, comoquiera que la realidad no se nos presenta parcelada, resulta absurdo $\longrightarrow$ tendenciosohacerlo nosotros, más allá de las necesidades metodológicas que instan a realizar el análisis desde "alguna" disciplina, "deficiencia" que debe ser matizada mediante la adecuada contextualización explícita de su ubicación.

9. La exposición más clara de esta posición aparece formulada en ROSTOW, W.W.; Las etapes del desarrollo económico, FCE, México, D.F, 1960.

10. Sobre este punto, pueden verse dos textos excelentes, por certeros y aclaratorios, ambos de Friedrich Engels. Se trata de Ludwig Feüerbach y el fin de la filosofia clásica alemana y Del socialismo utópico al socialismo científico (varias ediciones).

11. Es importante hacer notar que la teorla de la dependencia como tal no existe, pudiéndose hablar, en todo caso, de enfoque de la dependencia o de teorfas de la dependencia. Y dentro de éstas se encuentran unos análisis que toman como variable principal a las clases y otros que toman como tal a los países. Una tipología de ellas ayudaría a entender esto, si bien excede el alcance de este artículo. Al respecto, pueden consultarse, entre otros, los estudios siguientes: PALMA (1987), CUEVA (1979) , PAZ (1981) y GRIFFIN y GURLEY (1986).

12. En cualquier caso, resulta pertinente aclarar que insertamos el enfoque epistemológico - así como la metodología asociada a él- en un marco teórico de referencia marxista porque entendemos que debe ser crítico, materialista, dialectico, global e histórico, y no al revés. Es decir, no pensamos que ha de ser marxista y por ello le "adomamos" con esos rasgos. Dicho de otro modo: igual que resultaría absurdo optar por una opción "marxista" a priori, 
basada en alguna forma de "acto de fe", también lo resultaría obviar que un enfoque como el propuesto responde claramente a una matriz teórica de inspiración marxista. Aclaración ésta que, pensamos, no resulta gratuita.

13. MARX, Karl (1877); Russia's pattern of development, Letter to the Editorial Board of the Otechestyennige Zapiski, reimpreso en FEUER, L. (comp.); Marx and Engels: Basic Writings on Politics and Philosophy, Londres, Fontana Library, 1969. La cita está tomada de PALMA (1987; pág. 28).

14. PALMA (1987; pág. 29). Resulta interesante ver el final de la cita: "Su posición [la de Marx] con respecto al caso de Rusia ilustra bien la flexibilidad de su enfoque, que tiene su base en la unidad dialéctica de los factores subjetivos y objetivos. Stalin [Problems of Leninism, Moscú, Cooperative Publishing Society of Foreign Workers in the USSR, 1934, pág. 104] pervertirá posteriormente este enfoque, afirmando que el estilo soviético de dictadura del proletariado es la forma 'adecuada y obligatoria para todos los países sin excepción, incluso aquellos en una etapa de capitalismo desarrollado', condenando así a todos los países, excepto la Unión Soviética, a no tener su propia historia".

15. El materialismo histórico y el materialismo dialéctico como referentes epistemologicos y metodológicos no han aparecido explicitados hasta este momento por razones de claridad expositiva. Más concretamente, para poder presentar, en el apartado anterior, el análisis comparativo de los distintos enfoques con elementos más genéricos -histórico, dialéctico-. Precisamente son el materialismo histórico y el materialismo dialéctico los que realmente hacen requerir la declaración expresa de la adopción de la referencia marxista efechuada anteriormente.

16. TROTSKI, Lev (1930); Historia de la Revolución rusa, Editorial Sarpe, Madrid, 1985, tomo I, págs. 32 y 33.

17. Anticipando lo que vendrá a continuación, aprovechamos para aclarar que sólo existe un modo de producción capitalista, de manera que el hecho de que existan FSCD y FSCS no son sino expresión de distintas modalidades a través de las que tiene lugar dicho modo de producción, al tomar concreción en realidades históricas determinadas. Todo ello, al margen de la existencia de formaciones sociales donde el capital fue expropiado, las cuales, en todo caso, se insertan igualmente en una única economfa mundial hegemonizada por relaciones de tipo capitalista.

18. ENGELS, Friedrich; Carta a Sombart, 11-III-1895, en MARX, Karl y ENGELS, Friedrich; Obras escogidas, tres tomos, Progreso, Moscú, 1976, pág. 534. Tomada de PALAZUELOS (1986; pág. 23). El ejemplo más aberrante y, lamentablemente, más significativo de aquello que Engels señala como lo que no es la concepción de Marx es, sin duda, el estalinismo tanto en el plano económico como en el político. De ahd la falacia de su pretendido carácter "marxista". 